\title{
Médiévales
}

Langues, Textes, Histoire

54 | printemps 2008

Frères et sœurs

\section{Bernard de Clairvaux et la fratrie recomposée}

Bernard of Clairvaux and recomposed sibship

\section{Chloé Maillet}

\section{(2) OpenEdition}

Journals

Édition électronique

URL : https://journals.openedition.org/medievales/4483

DOI : $10.4000 /$ medievales.4483

ISSN : $1777-5892$

\section{Éditeur}

Presses universitaires de Vincennes

\section{Édition imprimée}

Date de publication : 1 juin 2008

Pagination : 13-34

ISBN : 978-2-84292-217-7

ISSN : 0751-2708

\section{Référence électronique}

Chloé Maillet, «Bernard de Clairvaux et la fratrie recomposée », Médiévales [En ligne], 54 I printemps 2008, mis en ligne le 10 septembre 2010, consulté le 22 avril 2022. URL : http://

journals.openedition.org/medievales/4483; DOI : https://doi.org/10.4000/medievales.4483

Ce document a été généré automatiquement le 22 avril 2022

Tous droits réservés 


\title{
Bernard de Clairvaux et la fratrie recomposée
}

\author{
Bernard of Clairvaux and recomposed sibship
}

\author{
Chloé Maillet
}

\begin{abstract}
Mon âme était attachée à la sienne ; et de deux elles n'en faisaient plus qu'une, non par la consanguinité, mais par l'harmonie. Certes, les liens charnels n'étaient pas absents, mais c'était plutôt l'affinité spirituelle, l'accord de nos âmes, la conformité de nos mœurs qui nous unissaient'.
\end{abstract}

1 La terminologie de la germanité et de la fraternité est encore aujourd'hui, plus que toute autre, propice à la désignation de relations non fondées sur la parenté biologique. Dans les textes hagiographiques médiévaux, qui vantent le mérite de la fraternité rituelle entre tous les chrétiens, le sens biologique n'est pas majoritaire derrière les appellations frater et soror. À tel point que lorsque l'on tient à préciser le lien du sang, les auteurs ajoutent souvent un adjectif comme germanus ou carnalis.

Comme l'avait montré Alessandro Barbero, l'écriture hagiographique est originellement fondée sur une tradition «d'abandon de la famille et de mépris absolu pour les raisons des parents $»^{2}$. Mais ce n'est pas pour autant que toute forme de référence à la parenté soit absente, bien au contraire. À la parenté charnelle s'oppose une pseudo-parenté ${ }^{3}$ de substitution qui lui emprunte sa terminologie. Elle est considérée comme supérieure, parce que fondée sur la caritas, l'amour parfait qui donne sa cohésion à l'ensemble de la société chrétienne ${ }^{4}$ appelée - . La valorisation de cette autre parenté est inversement proportionnelle à la dépréciation de la - parenté spirituelle première, considérée souillée par les liens de la chair. Le problème est que derrière le terme globalisant de parenté spirituelle se cache un ensemble de situations bien différentes. Il s'agit d'abord de la germanité de tous les chrétiens « frères dans le Christ » depuis le baptême, qui est le cadre de toute réflexion sur la parenté spirituelle au Moyen Âge. Il s'agit aussi, et c'est ce qui a fait l'objet du plus grand nombre d'études, de liens sociaux conventionnellement établis autour de ce même baptême: le parrainage et le compérage, qui entraînent des interdits de mariages spécifiques ${ }^{5}$. Il est enfin question d'une pseudo-parenté très particulière qui est celle dans laquelle vit une 
partie de la société médiévale, qui a choisi d'abandonner ses parents selon la chair au profit d'une famille spirituelle cléricale ou monastique, et c'est de ce cercle de parenté explicitement concurrent des parents charnels dont il sera question ici ${ }^{6}$.

Bernard de Clairvaux (1090-1153), le plus célèbre moine et abbé cistercien de la première moitié du douzième siècle, fut canonisé le 18 juin 1174 par le pape Alexandre III. Dans sa vie, la question des rapports entre parents charnels et spirituels se pose avec d'autant plus d'acuité qu'il choisit de ne pas abandonner sa fratrie biologique au seuil du monastère, mais de l'entraîner avec lui, faisant ainsi de ses frères et sœurs de chair des germains spirituels.

4 Le cas de saint Bernard est particulièrement intéressant pour aborder le lien adelphique par la radicalité de sa situation (il convertit tous ses frères et sœurs sans exception) et la multiplicité des sources qu'il permet de confronter. Nous ferons appel d'abord aux écrits de Bernard de Clairvaux, et en particulier ses sermons sur le cantique, soit conservés un grand nombre de manuscrits ${ }^{7}$, où il évoque à plusieurs reprises ses rapports avec certains de ses frères dans le monastère. Pour suivre la biographie de ces derniers, les vitae nous offrent un dossier hagiographique qui fut largement préparé du vivant du saint, souvent par ses proches. La Vita Prima ${ }^{8}$ fut rédigée par Guillaume de Saint-Thierry (c. 1085-1148) à partir des notes de Geoffroy d'Auxerre, son secrétaire et disciple. Son œuvre fut achevée après la mort de Guillaume par Arnaud de Bonneval. La première tentative de canonisation ayant échoué, on fit appel à un second hagiographe : Alain de Lille, évêque d'Auxerre ${ }^{9}$. La Vita Tertia est constituée des notes de Geoffroy d'Auxerre rassemblées par Mabillon. Ces textes eurent également une diffusion large, d'abord dans un contexte cistercien, puis grâce à leur insertion dans les compilations hagiographiques comme celle de la Légende dorée ${ }^{10}$. Nous commenterons également l'iconographie que ces épisodes de la vie de Bernard ont suscitée. Rappelons cependant que l'on ne possède aucune image exécutée du vivant du saint, hormis une hypothétique représentation sur un sceau ${ }^{11}$, et que son iconographie n'est pas aussi développée que sa notoriété pourrait le laisser supposer. Les images les plus fréquentes représentant Bernard de Clairvaux sont les portraits et deux scènes emblématiques, l'amplexu christum (miracle par lequel le Christ se serait détaché de la croix pour prendre Bernard dans ses bras) et la lactation par la Vierge ${ }^{12}$. Mais quelques scènes provenant souvent de légendiers enluminés peuvent nous renseigner sur l'histoire des représentations de la fratrie du saint, à l'époque où il était un saint vénéré et considéré comme un modèle pour de nombreux moines ${ }^{13}$.

Le refus de quitter sa parenté biologique

5 Avant d'être connu sous le nom de Bernard de Clairvaux, le futur saint fut d'abord Bernard de Fontaine, jeune noble élevé au sein d'une grande fratrie de six frères et une sœur. Son père Técelin (dit le Rouxou ou le Saur), seigneur de Fontaine-lès-Dijon était originaire d'une famille de la moyenne noblesse liée à d'importants groupes aristocratiques, comme les Châtillon, les La Roche Vanneau, et les vicomtes de la Ferté. Técelin était d'ailleurs un grand officier des ducs de Bourgogne et détenait le château de Châtillon pour le comte Henri II à l'époque où Bernard faisait ses études chez les chanoines de Saint-Vorles. Técelin contracta autour de 1180 un mariage hypergamique avec une femme de haute noblesse, Aleth de Montbart, du bourg du même nom ${ }^{14}$.

6 Cette dernière était la fille de Bernard de Montbard, un des optimates du duc de Bourgogne, dont le futur saint tenait probablement son nom. Les frères d'Aleth, Rainard, Milon et Gaudry détenaient le plateau de l'Armonçon, Semur-en-Auxois, et la 
haute vallée de la Seine. Toutes les vies de saint Bernard insistent sur l'influence cruciale qu'eut sur lui la dévotion de sa mère. Selon un thème hagiographique récurrent ${ }^{15}$, Guillaume de Saint-Thierry précise que sa mère « refusa toujours de faire nourrir ses enfants du lait d'un étrangère, comme si avec le lait maternel, elle dût les remplir de tout ce qui pouvait se trouver de bon en elle ${ }^{16}$ ». Il avait été très jeune dédié à une future vie monastique, envoyé étudier à l'école des chanoines de Saint-Vorles, peut-être sous l'influence de sa dévote mère. La mort de celle-ci, alors que Bernard n'avait que seize ou dix-sept ans semble avoir été une expérience très douloureuse. À vingt-et-un ans il rentra au monastère de Cîteaux fondé par Robert de Molesme en 1098.

7 L'histoire de la conversio de la parentèle de Bernard est bien connue. Elle est décrite dans sa vie comme un feu qui «s'attaquant de proche en proche à tout ce qui l'environne, finit par consumer même ce qui se trouve au-delà. Ainsi ce feu que le Seigneur avait envoyé dans le coeur de son serviteur, pour qu'il y allumât un incendie, s'attaque d'abord à ses frères, n'épargnant que le plus jeune d'entre eux, parce qu'il était dans un âge trop peu avancé encore pour prendre part au changement de vie de ses frères, et le plus âgé qui resta pour être la consolation de leur père, dévore ensuite ses primes, puis ses compagnons et ses amis, tous ceux qui pouvaient enfin concevoir l'espérance d'une conversion ${ }^{17}$. » Le premier à le suivre fut son frère Barthélemy, qui n'avait pas encore commencé sa carrière militaire. André fit plus de difficulté, mais se rangea finalement à l'avis de Bernard. Guy, l'aîné, était marié et se sépara de sa femme pour le rejoindre au monastère. Cette dernière, dénommée Elisabeth se fit aussi moniale et devint ensuite prieure dans le monastère bénédictin de Jully-lès-Nonnains. Gérard et André, les deux derniers frères, finirent par se rendre à ses imprécations. Après la mort d'Aleth, Técelin rejoignit ses fils qui l'avaient délaissé pour le monastère. Le corps de la mère défunte, d'abord enterré à l'église Saint-Bénigne, rejoignit Clairvaux, pour achever l'absorption complète du premier cercle de la parentèle de saint Bernard. "Alors qu'il prêche en privé et en public, les mères cachent leurs fils, les femmes retiennent leurs maris, les amis se détournent mutuellement ${ }^{18}$. " Il voit en chacun de ses proches un moine potentiel. Il emmène avec lui de nombreux cousins dont Geoffroy de la Roche-Vanneau, apparenté à son père, qui deviendra évêque de Langres, Robert de Châtillon, qui trahit un temps son ordre pour Cluny, avant de fonder l'abbaye cistercienne de Noirlac, et surtout Gaudry de Touillon, son oncle, qui se sépare de sa femme entrée au couvent des moniales de Molesme.

Saint Bernard refuse ainsi de passer par une étape considérée essentielle avant toute entrée dans la vie monastique : quitter sa parenté ${ }^{19}$. Tous ses frères vinrent avec lui à Cîteaux. Les premières terres qui furent données pour les nouvelles fondations de l'ordre, alors en pleine expansion, provenaient de dons de son père et d'autres de ses cousins $^{20}$. Et il continua à entretenir des relations avec ses parents restés en dehors du monastère : citons par exemple le fait qu'il dédia un texte à la nouvelle chevalerie, les Templiers ${ }^{21}$, dont son oncle, André de Montbard (1103-1156), était l'un des fondateurs, et devint le cinquième maître de l'ordre.

9 Entre frères et sœurs de la noblesse, le principal conflit qui puisse s'imposer concerne l'héritage, surtout à une époque où, bien que la primogéniture ne soit pas toujours la règle, les cadets restent défavorisés. Le seul épisode de la vie de saint Bernard concernant les problèmes de concurrence entre frères fut repris dans les trois vitae et jusqu'à la Légende Dorée de Jacques de Voragine ${ }^{22}$. 
Guy, l'aîné de tous, apercevant Nivard, le plus jeune de leurs frères, qui était encore enfant et se tenait dans la cour de la maison avec d'autres enfants, lui dit : « Allons, Nivard, tous nos biens sont à toi maintenant. » À ces mots, l'enfant répondit d'une manière peu enfantine : "Ainsi, vous prenez le ciel et vous me laissez la terre ; le partage n'est pas égal. » Après avoir échangé ces paroles, ils s'éloignèrent ; quant à Nivard, il resta à la maison avec son père, mais peu de temps après il alla rejoindre ses frères ; il n'y eut ni père, ni proches, ni amis qui purent le retenir. Il ne restait donc plus de toute cette famille consacrée à Dieu, que le père déjà vieux, avec la fille dont nous parlerons aussi en son lieu ${ }^{23}$.

Dans cet épisode de la vie du saint, conçu comme une anecdote exemplaire, la concurrence entre les frères est niée. Le patrimoine familial fut négligé par chacun des frères. L'épisode est d'autant plus frappant que c'est Guy, l'âné, qu'avait mené une vie laïque avant de prendre l'habit, lequel qui annonce son refus d'hériter au benjamin Nivard. L'inversion est double. L'aîné propose son héritage au benjamin, et celui-ci replace immédiatement la concurrence dans un système spiritualisé : l'accès au ciel ${ }^{24}$.

11 La conversion de ses frères par Bernard pourrait presque être lue comme une revanche du cadet (soumis à la vie monastique) entraînant, par sa force de conviction insistante, tous ses frères avec lui. Il replace du même coup la fratrie dans une égalité face à l'héritage et inverse ainsi les valeurs nobiliaires dans lesquelles la parenté s'était constituée. Et c'est bien la grande particularité de l'écriture hagiographique que de présenter la germanité, un lien par définition hiérarchisé selon l'ordre de naissance, comme un modèle d'égalitarisme. Précisons que l'ensemble de la fratrie ayant été convertie à Cîteaux, il s'agissait non seulement d'un héritage spirituel mais aussi matériel. Técelin avait lui aussi fait don de ses biens à l'abbaye quand il s'y retira.

Bernard et la «transgermanité $»^{25}$

"Bernard quitta le toit, suivi de ses frères dont il était devenu le père et qui se regardaient comme ses enfants spirituels, puisqu'il les avait engendrés au Christ par la parole de $v^{2} e^{26}$.» La parenté spirituelle a la particularité de permettre une superposition des liens de parenté, qui serait impossible ou incestueuse dans le domaine de la parenté charnelle ${ }^{27}$. Dans le contexte cistercien, ces métamorphoses de la parenté sont associées à une grande valorisation des métaphores d'engendrement. La conversion était pensée comme une seconde naissance, dans laquelle Bernard se considère géniteur : "C'est moi qui t'ai engendré à la religion par mes leçons et mes exemples ; c'est moi encore qui t'ai nourri de lait ; [...] tu as été enlevé de mon sein ", écrit Bernard à son cousin Robert de Châtillon enfui à Cluny ${ }^{28}$. "Montrez-vous comme des mères quand vous réconfortez, comme des pères lorsque vous corrigez ${ }^{29}$ ", écrit-il dans ses sermons. En tant qu'abbé, Bernard alterne entre figure paternelle et maternelle. Mais il nous semble que c'est souvent le lien adelphique qui l'emporte, qui se surajoute aux deux précédents ${ }^{30}$. Un sondage lexical dans le texte de la Vita prima Bernardi nous montre que les termes désignant des liens de germanité (frater, soror, germanus, germana) arrivent en première place des termes désignant la parenté primaire avec $43,9 \%$ des occurrences ${ }^{31}$. À titre de comparaison, on notera que dans une compilation hagiographique comme la Légende Dorée de Jacques de Voragine, comprenant un remaniement de la Vita prima parmi ses 178 chapitres, les liens de germanité ne se placent qu'en troisième position, après la paternité et la filiation, avec $20,9 \%$ des occurrences ${ }^{32}$.

Cette surreprésentation des liens de germanité est liée, dans la vie de Bernard, aussi bien à la vie monastique, qu'aux liens de sang qu'il entretenait avec certains moines. 
C'est cela qui entraîne à la fois une valorisation forte de ce lien mais aussi une grande confusion apparente dans les personnes désignées : qui sont les frères spirituels, et qui sont les frères charnels? Une simple étude terminologique ne permet pas de le définir strictement, parce que souvent les statuts se recoupent. Cette ambiguïté des termes n'est pas seulement une convention de langage. Elle représente bien plutôt une ambiguïté et parfois une ambivalence volontaire de terminologie classificatoire : dans le cas des frères de Bernard, ils sont tous à la fois des frères charnels et des frères spirituels. Ce système complexe de désignation des liens de germanité possède en quelque sorte un équivalent en images.

En effet, dans certaines images, la synthèse entre paternité de l'abbé et germanité du moine s'exprime parfois plus efficacement que dans les textes. L'on trouve souvent dans les manuscrits hagiographiques l'image paternelle de l'abbé sermonnant ses moines ${ }^{33}$. Prenons l'exemple d'une image-frontispice de 1303, présente au premier folio d'un manuscrit des Sermons sur le cantique (fig. 1).

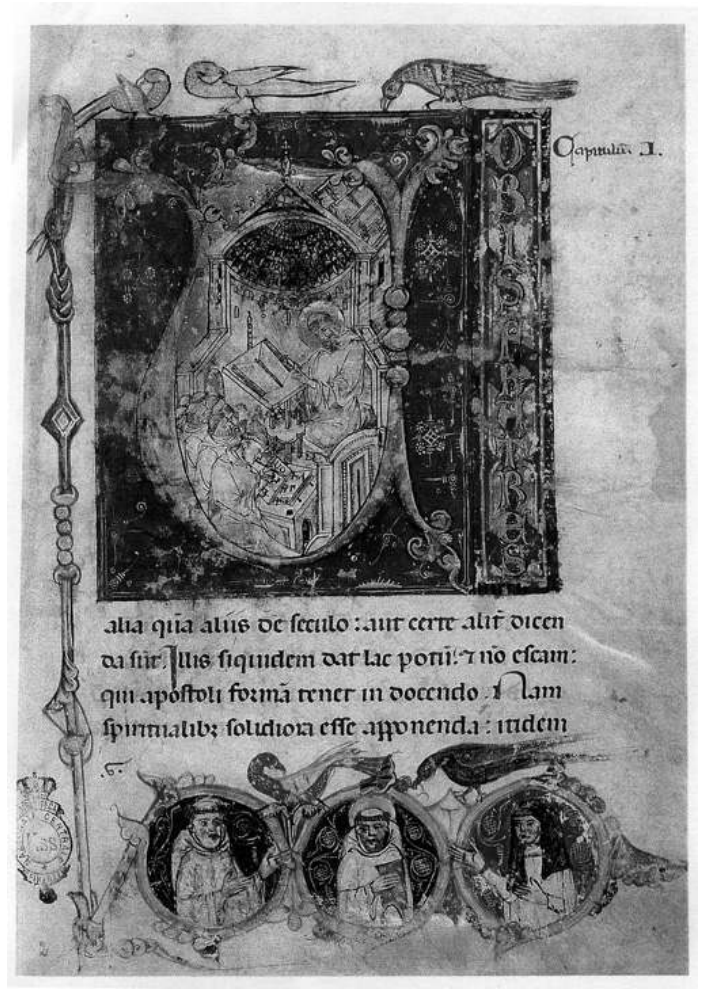

Fig. 1 : Bernard de Clairvaux, Sermones super Cantica, Florence, Biblioteca Nazionale Centrale, conv. Soppr. J.VI.22, f. 1v, Italie du Nord (Bologne ?), 1303 (Publié dans L. DAL PRÀ (dir.), Bernardo di Chiaravalle nell'arte italiana dal xiv al xvIII secolo, Milan, 1990).

Dans une initiale historiée dorée sur fond bleu cobalt occupant plus de la moitié de la page, est représenté l'intérieur d'une salle capitulaire de monastère. Sept moines sont figurés massés dans la courbe gauche du « U», l'un d'entre eux prenant des notes alors que les autres prient. On a l'impression que tous les moines ne font qu'un corps. Cela est accentué par un détail stylistique : les personnages et objets sont figurés légèrement vus du dessus, ce qui rappelle la vue que l'on peut avoir des salles capitulaires cisterciennes depuis le cloître, souvent construites quelques marches en contrebas. Occupant la plus grande partie de l'image, Bernard est inscrit avec amplitude dans l'espace, dans une position dominante, s'ouvrant sur la coupole. Le saint appartient au groupe de moines de l'initiale tout en les dominant. Nous avons ici à la fois l'image 
d'une paternité spirituelle de l'abbé et de la fraternité étendue des moines, dans laquelle ce dernier s'inclut. Le lien entre frères et père est souligné d'autant que Bernard n'est pas seulement un abbé, il est un saint. Au moment où est peinte cette image, un siècle et demi après sa mort, il est considéré comme le second père fondateur de l'ordre.

16 L'initiale historiée s'ouvre aussi sur des rinceaux hybrides végétaux et animaux, qui descendent jusqu'au bas de la page pour venir dessiner trois médaillons entrelacés. Le médaillon central propose une deuxième image du saint nimbé portant un livre à la main. Les deux autres représentent un moine et une moniale. Les trois médaillons sont de même taille et reliés les uns aux autres par des rinceaux. Le moine et la moniale sont tournés vers le saint les bras ouverts. Ils représentent des figures tutélaires des branches féminines et masculines de l'ordre, et offrent ainsi une image du lien horizontal qui unit ces abbayes-sœurs ${ }^{34}$.

17 En revanche, les représentations de saint Bernard accompagné de ses frères et sœurs " selon la chair » ne sont pas très fréquentes au Moyen Âge. On connaît cependant un polyptyque des années 1365, conservé à l'Académie de Florence qui les représente (fig. 2).

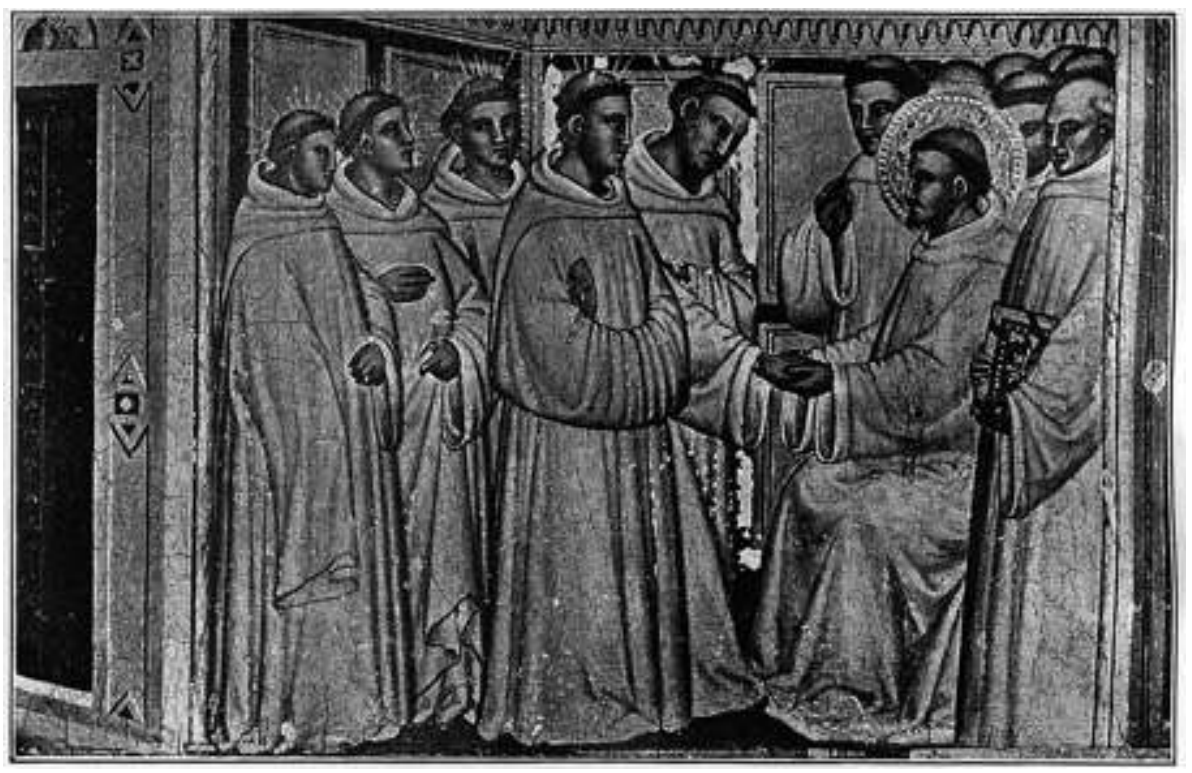

Fig. 2 : Matteo di Pacino (Maître de la chapelle Rinunccini), Vision de saint Bernard (détail), Florence, Galleria dell'Accademia, c. 1365

Ce polyptyque est constitué d'un panneau central représentant l'apparition de la Vierge à saint Bernard, et d'une prédelle représentant d'un côté le saint prêchant contre les hérétiques à Sarlat et de l'autre, le saint au milieu de ses frères/moines ${ }^{35}$. L'image principale figure le saint dans une relation de filiation par rapport à la Vierge, typique de la mariologie bernardine. La prédelle le représente dans une position dominante de père prédicateur. Celle qui nous intéresse ici met en valeur des liens plus horizontaux. Bernard siège au milieu des moines, ses frères spirituels placés légèrement en retrait par rapport à lui. Face à lui, cinq moines que l'on peut identifier comme ses frères de sang sont auréolés de lumière. Un seul se détache en avant, et se distingue par une plaie christique au côté. Il s'agit donc de celui qu'il a maintes fois désigné comme son frère le plus cher, Gérard. Il lui avait prédit une plaie au flanc s'il refusait la conversion. Celui-ci fut, selon la légende, réellement blessé lors d'une 
campagne militaire, et décida alors de rejoindre son frère à Cîteaux en 1112, avant de l'accompagner à Clairvaux (fondée en 1115).

C'est à son frère Gérard que Bernard consacra l'un de ses sermons les plus célèbres, le vingt-sixième des Sermons sur le Cantique. On sait que Gérard, très proche de Bernard, était cellérier de l'ordre. Il fut tant célébré à Clairvaux qu'un culte local lui fut rendu à sa mort. Il accompagna Bernard dans son troisième voyage en Italie en avril 1137, et tomba là-bas gravement malade. Bernard pria pour sa guérison. Quand elle survint, elle fut considérée comme un miracle ${ }^{36}$. Il mourut peu de temps après son retour à Clairvaux, sans doute autour de 1139, alors qu'il n'avait pas cinquante ans.

C'est une véritable oraison funèbre en même temps qu'un traité sur les parentés charnelles et spirituelles que Bernard inséra dans ses sermons. La singularité de ce texte explique qu'il se soit très tôt diffusé, et ait suscité de nombreux commentaires du vivant même de son auteur. Il fit l'objet d'une critique virulente par Béranger de Poitiers, le disciple et défenseur d'Abélard : « Nous pourrions accepter les explications de notre auteur disert, s'il n'avait composé une tragédie plutôt qu'un commentaire. Car quand on ouvre son livre à certaines pages, il mentionne de façon inattendue la mort de son frère, pour les funérailles duquel il écrit presque deux cahiers ${ }^{37}$. »

Bernard écrivait : "Qu'ai-je à faire de ce cantique, moi qui suis dans l'amertume ? La douleur cuisante détourne mon attention ${ }^{38}$. » La douleur qu'il éprouve à la mort de son frère l'amène ainsi à un commentaire sur les liens adelphiques charnels et spirituels : "Il était mon frère par le sang, mais plus encore par la vocation religieuse ${ }^{39}$."

Il est intéressant de suivre avec précision le déroulement de son raisonnement. Dans une première partie, Bernard bat sa coulpe. Il spiritualise sa douleur en expliquant qu'elle provient d'une immixtion des âmes (homo unanimis), lien indissoluble comme celui du mariage grégorien, qui faisait correspondre la séparation à un divorce : « 0 , séparation très amère, que seule la mort pouvait effectuer, vivant, m'aurais-tu délaissé ma vie durant? Cet horrible divorce ne pouvait être que l'œuvre de la mort. Qui donc n'aurait épargné le lien si doux de notre amour mutuel, sinon la mort, ennemie de toute tendresse ? Oui, vraiment, c'est la mort qui, en enlevant l'un de nous, dans sa fureur a fait périr les deux. N'est-ce pas la mort pour moi aussi ? ${ }^{40} »$. Au troisième chapitre, nous restons dans la description d'un abandon : «Tu connais la situation où je croupis, où tu m'as abandonné : personne qui me tende la main ${ }^{41}$. " Le quatrième chapitre décrit avec plus de précision la nature de leur lien. Gérard, s'occupant des affaires matérielles, laissait Bernard libre de se consacrer aux choses de l'esprit. Il fait ainsi référence à une image adelphique fameuse: le modèle des deux sœurs évangéliques Marthe et Marie. Bernard ne les cite pas, mais c'est ainsi que glose le Grand exorde de Cîteaux ${ }^{42}$. Il cite aussi les dernières paroles de Gérard, qui furent reprises dans le Grand exorde : "Mon Dieu, tu sais que dans la mesure de mes possibilités, j'ai toujours désiré la paix, pour m'occuper de mon âme et pour vaquer à toi, Seigneur. Mais j'étais impliqué par la crainte de toi, la volonté des frères, le désir d'obéir, et surtout l'amour fraternel (germana dilectio) pour celui qui était à la fois mon abbé et mon frèr $\mathrm{e}^{43}$. » Le chapitre $5 \mathrm{du}$ texte énumère toutes les qualités et les fonctions de Gérard, et ce en quoi Bernard lui était redevable. Mais il s'interrompt et dit : «Je parle à des gens qui savent tout cela (Rom. 7, 1), et qui pourraient en dire encore plus à ce sujet. Je me retiens cependant, "parce qu'il est mon frère et ma chair" (Gen. 37, 27) ${ }^{44}$. "

23 Le dernier chapitre est une synthèse et une auto-justification. Selon Bernard, ce n'est pas le lien du sang qui fait sa proximité avec Gérard. «Je pleure Gérard, c'est Gérard qui 
est en cause, mon frère selon la chair, mais l'homme le plus proche de moi selon l'esprit, mon compagnon dans la poursuite du même but. Mon âme était attachée à la sienne ; et de deux elles n'en faisaient plus qu'une, non par la consanguinité, mais par l'harmonie. Certes, les liens charnels n'étaient pas absents, mais c'était plutôt l'affinité spirituelle, l'accord de nos âmes, la conformité de nos mœurs qui nous unissaient ${ }^{45}$. " On voit bien ici combien la parenté charnelle ne se rachète que parce qu'elle est spiritualisée, améliorée par l'union de l'âme ${ }^{46}$. Et cette parenté est à la fois calquée sur celle du corps, mais bien différente dans la catégorisation des liens et de leurs possibles superpositions : «Gérard était à moi, oui, à moi. Comment n'aurait-il pas été mien, lui qui fut mon frère par le sang, mon fils par la profession religieuse, mon père par la sollicitude, mon compagnon par l'accord de nos esprits, mon intime par l'affection? Il m'a quitté : je le sens, je suis blessé, et ma blessure est grave ${ }^{47}$. »

Dans ce sermon, Bernard et Gérard passent par tous les liens de parenté : matrimonial d'abord, fraternel ensuite et paternel finalement. Devenant père de son frère, il s'affirme aussi comme étant son fils, pour relativiser la position dominante sur tous les frères de sa position d'abbé. Il en vient ainsi au plus haut degré d'immixtion et d'ambiguïté dans les catégories des liens de parenté. N'oublions pas qu'il était aussi le père et frère de son propre père, ce dernier ayant fini ses jours dans le monastère. La pseudo-parenté monastique aplanit les liens, les «horizontalise » à l'extrême puisque tous les hommes chrétiens sont frères. Mais cet aplanissement va aussi de pair avec une exaltation d'un lien plus vertical, la paternité qui lie de manière pyramidale les moines à un même père, l'abbé.

La complexité exemplaire des liens dans la parentèle de saint Bernard n'avait pas échappé aux enlumineurs qui choisirent parfois ce thème pour illustrer la vie du saint. On peut prendre l'exemple de deux images présentes dans des manuscrits de la fin du quinzième siècle : le Miroir Historial du musée Condé (fig. 3) et la Légende Dorée de la Bibliothèque nationale de France (fig. 4) et, dont on pense que les miniatures ont été exécutées par des artistes parisiens assez proches l'un de l'autre ${ }^{48}$. 


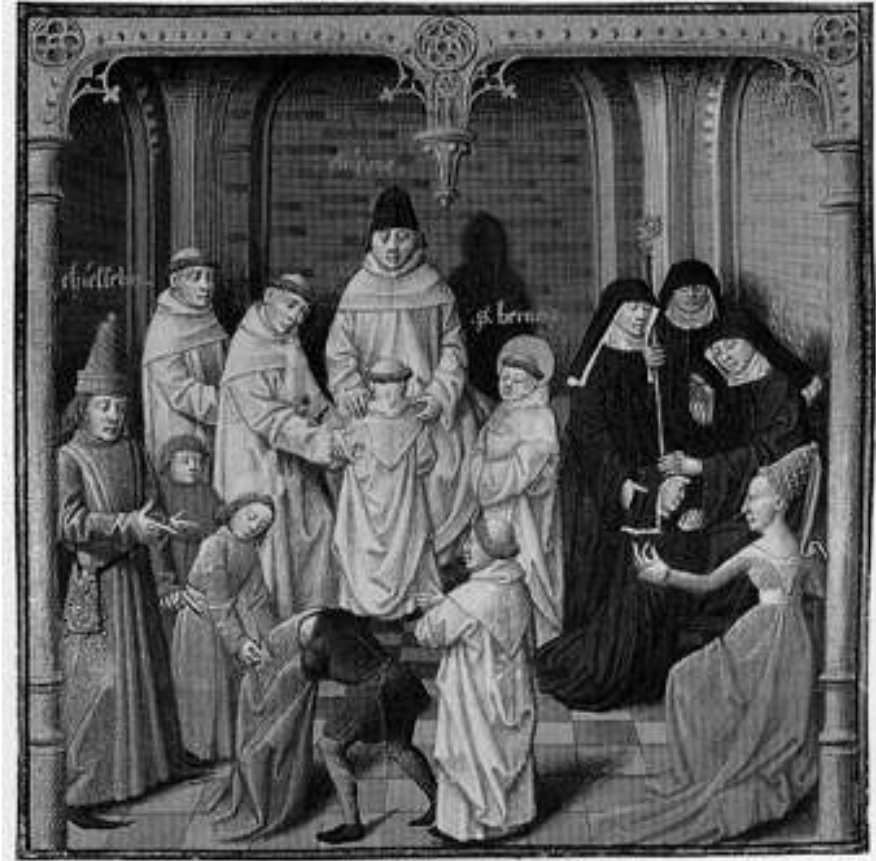

Fntrée de Bernard a Citeaux. Vincent de Beauvais, Miroir historial, xv' siècle. Chantilly, Muséc Condé, Ms. 722, folio 209 v (cl. Giraudon)

Fig. 3 : Vincent de Beauvais, Miroir historial, Musée Condé, Ms. 722, f. 210, Maître François, Paris, C. 1470-80 (Publié dans M. Pastoureau et G. Duchet-suchaux, La Bible et les saints, Guide iconographique, Paris, 2006).

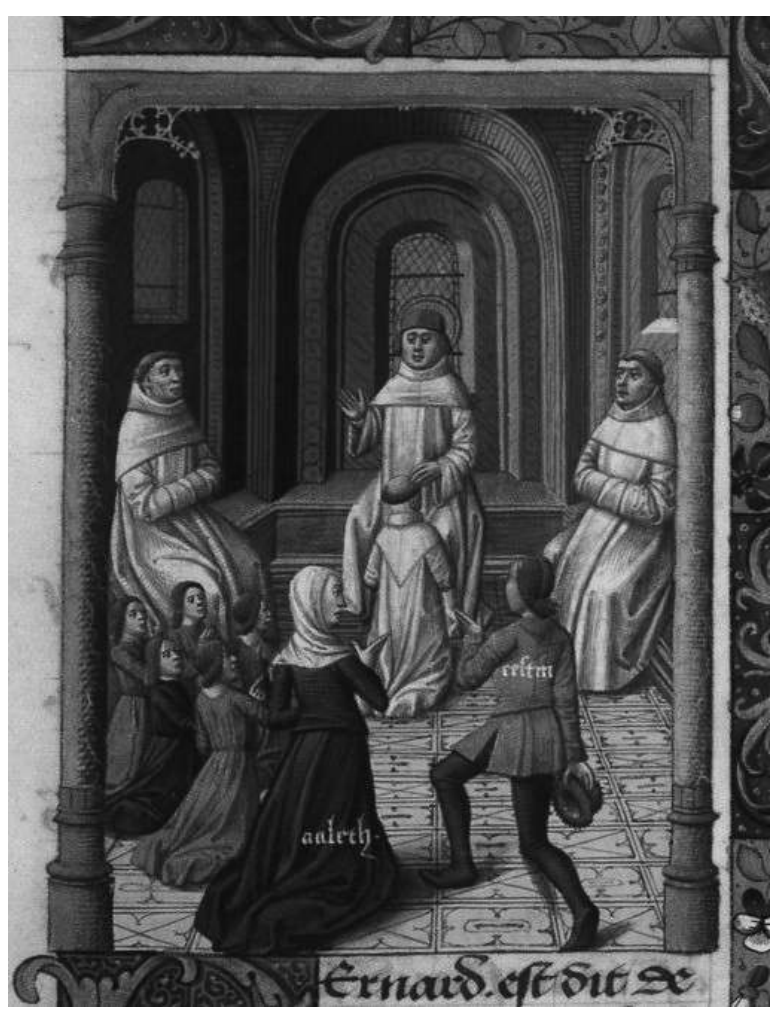

Fig. 4 : Jacques de Voragine, Légende Dorée, BNF, Fr. 245, f. 55, Maître de Jacques de Besançon, Paris, c. 1480-90 (Publié sur www.mandragore.bnf.fr). 
Miroir historial représente l'entrée de Bernard et de ses frères et sœur à Cîteaux en figurant trois groupes de personnages distincts. À gauche, autour de Técelin, trois garçons sont présentés en habits laïcs, l'un d'entre eux marquant le passage d'un état à l'autre en enfilant son habit ${ }^{49}$. À droite de l'image se trouve le groupe des femmes, au sein duquel Aleth offre sa fille aux moniales. Remarquons que le nom d'Aleth n'est pas inscrit sur l'image comme celui de Técelin, et que les femmes sont placées à gauche de l'abbé, dans la partie la moins valorisée de l'image. L'image est latéralisée selon des critères de genre. Au centre, l'abbé Étienne Harding accueille entre ses bras un jeune moine (sans doute un frère de Bernard), tandis que le saint nimbé est déjà placé à sa gauche. Chacun des groupes de personnages est construit sur une structure pyramidale que surplombe une figure paternelle (Étienne Harding au centre, Técelin à droite) ou maternelle (l'abbesse), mais l'ensemble de ses groupes est dominé par la figure imposante de l'abbé. L'image du lien de germanité entre les frères de sang et les moines est mise en valeur par leur petite taille qui les place sur un pied d'égalité. L'image de la Légende dorée rend la structure de double inclusion plus visible encore, plaçant au sommet de la pyramide la figure tutélaire de Bernard, reconnaissable à son nimbe. L' « horizontalisation » de son lien avec les moines est marqué par le fait que ces derniers sont assis à la même hauteur, tandis que le lien vertical de paternité s'exprime par sa position surplombante et par sa grande taille. Elle est figurée aussi par le fait que, comme Étienne Harding dans l'image précédente, il accueille entre ses bras un jeune moine. Aleth et Técelin sont plus petits que Bernard (ils sont ses enfants) mais plus grands que leurs autres enfants représentés à leurs côtés, leurs cinq fils probablement, qui rejoindront par la suite le monastère. Ces deux exemples sont ainsi deux tentatives de transposition en images de cette " parenté-gigogne » qui font que l'on peut être père de son propre père, et frère de ses enfants.

Mais ces images, peintes longtemps après que Bernard a rejoint le grand cortège des mythes hagiographiques, réinterprètent en fonction des outils iconographiques qui sont les leurs, et s'inscrivent dans une revalorisation des larges parentèles. C'est à cette époque que se développe, suite à la vision de sainte Colette en 1406, l'image de la sainte parenté de la Vierge, et du triple mariage de sainte Anne avec ses petits groupes ternaires de parents ${ }^{50}$. Elles ne correspondent pas non plus à l'historique de la conversion des frères, qui ne s'est pas faite de manière collégiale. Un membre de la fratrie a résisté plus que les autres à cette conversion, c'est sa sœur Hombeline.

Frère et sœur, passion secrète ${ }^{51}$

La relation entre saint Bernard et son unique sœur Hombeline est, dans les vitae, racontée bien après l'histoire de la conversion de ses frères. L'épisode principal est celui d'une visite qu'elle a rendue à ses frères qui refusent de la rencontrer :

Leur sœur, qui était restée dans le monde où elle s'était mariée, y menait une vie toute mondaine, au milieu de tous les dangers qui accompagnent les richesses de la terre ; un jour pourtant, Dieu lui inspira la pensée d'aller voir ses frères ; mais lorsqu'elle fut arrivée pour voir son vénérable frère, et qu'elle attendait avec une suite nombreuse et magnifique qu'il vînt recevoir sa visite, Bernard ne lui témoigna que de l'horreur et une sorte d'aversion comme pour une personne qui aidait ellemême le démon à dresser des pièges aux âmes pour les prendre, et il ne voulut point se montrer à elle pour recevoir sa visite. En apprenant son refus, cette femme se sentit toute couverte de confusion, et, profondément affligée de voir qu'aucun de ses frères ne daignait se déranger pour la recevoir, elle ne put s'empêcher de fondre en larmes en entendant les reproches que son frère André, qu'elle avait trouvé à la 
porte du monastère, lui adressait au sujet du luxe de vêtements dont elle environnait le fumier de son corps ${ }^{52}$.

Hombeline promet alors de suivre tous les conseils qu'il lui donnera si Bernard daigne la rencontrer. Il lui recommande alors de vivre selon les préceptes de l'Église et d'abandonner tout culte de l'apparence. Elle suit « religieusement » ses conseils au point de «mener la vie d'une ermite dans le monde ». Jusqu'au jour où son mari, devant ses demandes répétées, accepte qu'ils se séparent. «Profitant donc de la liberté qu'elle avait si longtemps désirée, elle se rendit au monastère de Jully, et y consacra à Dieu le reste de sa vie, parmi les saintes femmes qui s'y trouvaient déjà réunies. Là, le Seigneur lui fit la grâce de l'élever à un tel degré de sainteté, qu'elle montra bien, non moins par l'âme que par le corps, qu'elle était sœur de tous ces hommes de Dieu ${ }^{53}$.» Elle suivit si bien les conseils de son frère qu'elle devint même prieure de Jully, juste après Elisabeth, sa belle-sœur, épouse de Guy. Car c'est un prieuré de bénédictines, et non un monastère cistercien que Bernard recommanda à sa sœur ; preuve supplémentaire de la différence de traitement qu'il marque entre ses frères et sa sœur ${ }^{54}$. Cet épisode se présente donc comme une variation sur le thème de la pécheresse repentie à laquelle elle s'identifie elle-même: "C'est précisément parce que je suis pécheresse que je recherche l'entretien des saints, et si mon frère méprise mon corps, que le serviteur de Dieu ait pitié de mon âme ${ }^{55}$. » Cet épisode du rendez-vous refusé puis accordé fut mis en images à plusieurs reprises, notamment dans le Ci-nous dit, recueil d'exempla enluminé vers $1330^{56}$. Celui-ci synthétise l'épisode en trois affrontements de couples adelphiques. Un moine (son frère André) est présenté face à saint Bernard (pour lui demander s'il recevra sa sœur). André se retrouve face à Hombeline vêtue en aristocrate et lui signifie de la main son refus. Le dernier couple est celui de la réconciliation : Hombeline vêtue en quasi-moniale, corps et tête couverts, est représentée face à saint Bernard. Un autre manuscrit, datant du deuxième quart du quatorzième siècle, connu sous le nom de Légendaire angevin-hongrois, illustre également cet épisode, en y ajoutant une scène inédite (fig. 5) $)^{57}$. 


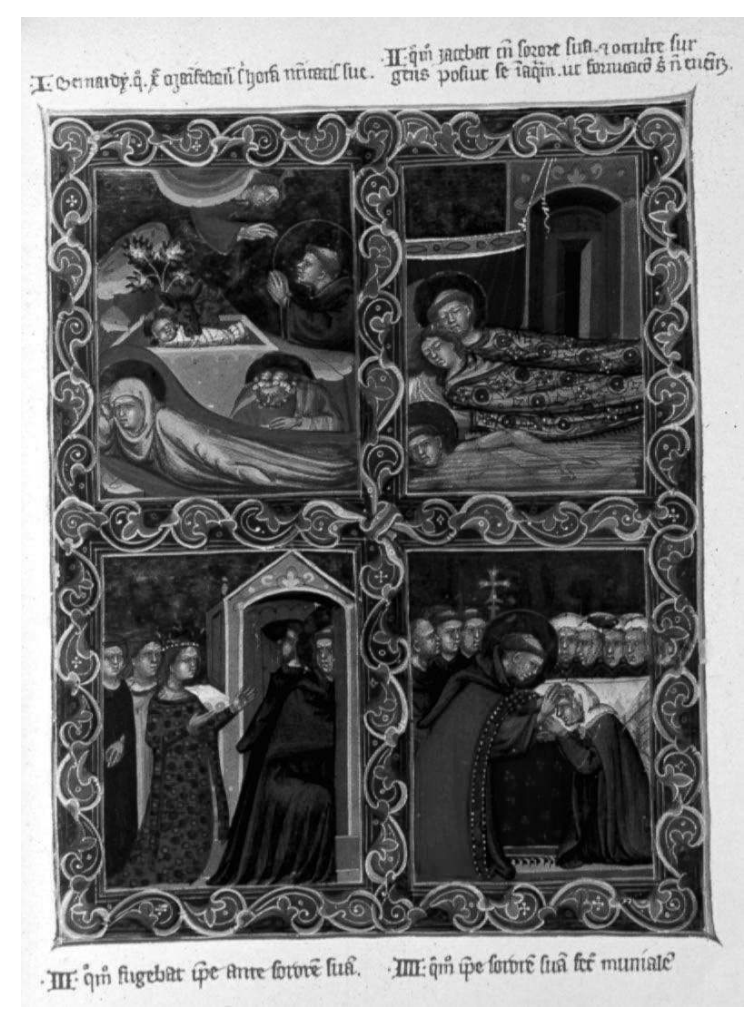

Fig. 5 : Légendaire Angevin Hongrois, B.A.V. Vat. Lat. 8541, f. 88v, Bologne?, c. 1325-50. pour seule indication textuelle une ligne de texte au-dessus de chaque image. Les deux scènes du registre inférieur représentent la même scène que celle du Ci nous dit. La séparation entre le frère et la sœur est plus marquée que dans cette dernière par la présence d'une architecture autour des moines renvoyant au monastère auquel la jeune femme richement vêtue ne peut accéder. Si la vision de la - l'identification de la première image ne fait pas de doute la deuxième saynète est plus équivoque. - nativité du Christ par saint Bernard On y voit le saint dans un intérieur, allongé au lit avec une femme. Juste en dessous, il est à nouveau représenté (on le reconnaît à son nimbe) allongé dans les eaux d'une rivière. Cette scène rappelle un épisode de sa vita : «En effet, un jour que, dans un mouvement de trop grande curiosité, il avait arrêté pendant quelque temps les yeux sur une femme, à peine rentré en lui-même, il rougit de soi, au fond de son coeur, se charge de se punir lui-même sévèrement de sa faute, et va se précipiter jusqu'au cou dans un étang glacial, qui était près de là ; il y demeure jusqu'à ce que, pâle de froid, il ait senti, par un effet de la grâce coopérante, le feu de la concupiscence charnelle se glacer en lui ${ }^{58}$.» Mais la légende du texte explique sans ambiguïté que cette image le représente couché avec sa sœur, et que pour éviter la fornication, il se jeta dans l'eau ${ }^{59}$. L'épisode de la sœur tentée par les richesses du monde s'est ici doublé d'une image d'elle en femme tentatrice ${ }^{60}$.

31 La relation de Bernard avec son unique sœur peut sembler à première vue proche de celle qu'il a eue avec ses frères. Elle fut convertie à l'habit monastique, après avoir renoncé à son mariage, tout comme Guy, dont elle retrouve l'épouse dans son prieuré. Pourtant, dans le texte sur sa conversion, racontée dans un autre chapitre de la vita que celui de ses frères, comme dans son iconographie, le conflit et la tentation des valeurs terrestres (richesses, vêtements, mariage, concupiscence), sont davantage mises en 
évidence. Bernard choisit d'ailleurs de l'orienter vers une abbaye bénédictine, et non cistercienne. Le lien frère-sœur se distingue catégoriquement du lien entre frères. Hombeline est la sœur, une femme vouée de toutes façons à ne pas embrasser le même genre de vie que le reste de la fratrie.

Ce parcours au sein de la fratrie du père spirituel des cisterciens présente à la fois des caractéristiques exemplaires, par la façon précise dont il dessine les contours de la parenté spirituelle, et des spécificités, liées à la situation et sans doute à la personnalité singulière de l'abbé. Elevé au sein d'une large fratrie nobiliaire, Bernard est un cadet qui par sa force de persuasion, relevée par tous ses contemporains, parvint à ce que ses frères et sœurs renoncent aux attraits de la vie chevaleresque et maritale. Cette influence d'un moine fervent au sein d'une fratrie n'est pas rare au XII siècle. Pierre le Vénérable eut par exemple trois frères, Jourdan, Pons et Armand, qui furent respectivement abbé de Chaise-Dieu, de Vézelay et de Maglieu. Son autre frère Héracle devint archevêque de Lyon. Abélard, l'ennemi de saint Bernard, eut lui aussi un frère chanoine de la cathédrale de Chartres, Porchaire. Mais dans le cas de Bernard, la fratrie refusa de se scinder entre différents ordres. Selon la Vita tertia, "André dit à Bernard: faites en sorte qu'aucun de nos frères ne reste dans le siècle, autrement il faudrait le couper par la moitié, car il ne saurait vivre séparé ni de vous ni d'eux ${ }^{61}$. B Bernard recréa ainsi au sein du monastère une parenté spirituelle qui non seulement était constituée sur le modèle de sa parenté charnelle, mais qui se superposait aussi exactement à celle-ci.

Il nous montre aussi comment le lien adelphique caractérisé par sa hiérarchie selon l'ordre de naissance devient par sa spiritualisation un lien horizontal, égalitaire. L'intérêt des écrits qu'il nous a laissés évoquant les juxtapositions des liens de parenté est essentiel pour la compréhension du fonctionnement de la parenté monastique à une époque réformiste. L'écart qui se redéfinit entre clercs et laïcs est essentiellement marqué par le refus ou l'acceptation du mariage qui devint un sacrement en 1181. Parce que les liens de germanité sont dénués de toute référence à la sexualité, ils sont les plus facilement "spiritualisables", et peuvent se conjuguer à l'amitié et à la caritas. La fusion de la germanité avec la paternité, et le ré-engendrement spirituel semblent avoir purifié ces liens, dont Bernard " euphémise " systématiquement le caractère charnel.

L'enchevêtrement des liens de filiation et de germanité qui font la spécificité de cette parenté spirituelle a parfois trouvé leur forme la plus accomplie dans des images, qui parviennent par des constructions inclusives et hiérarchisées, à rendre compte du lien de filiation, qui lie Técelin à ses fils, et du lien supérieur qui lie Bernard abbé à tous les membres de sa parentèle.

35 La question est différente lorsqu'il s'agit de la relation entre Bernard et Hombeline. Raymond Jamous, qui étudia le lien frère-sœur dans le nord de l'Inde (chez les Meo), remarque le fait que dans ce type de relation, le lien est à la fois celui d'une parfaite identité et d'une différence absolue, contrairement à celui qui unit les frères qui comporte uniquement la première caractéristique, et à celui qui relie mari et femme qui ne contient que la deuxième ${ }^{62}$. Comme l'écrit aussi Françoise Héritier, le lien frèresœur est celui « dans lequel l'identité des frères devient différence ${ }^{63}$. » La différence de genre entre les deux germains accentue l'écart bien mis en valeur par l'iconographie de la rencontre avec Hombeline à Cîteaux, et sa transformation vestimentaire, pensée comme une métamorphose spirituelle. Cela nous montre combien le lien adelphique 
doit être pensé en fonction du genre des germains qui est un caractère distinctif essentiel.

Dans les œuvres de Bernard de Clairvaux, les liens du sang restent centraux, et le sermon singulier que Bernard écrit à son frère le montre. Ce sermon s'inscrit dans la filiation de l'oraison funèbre prononcée par saint Ambroise pour son frère Satyrus, auquel il a été comparé. Mais il montre davantage qu'entre les frères spirituels, certains sont plus importants que d'autres. Ce sermon est beaucoup plus long et plus lyrique que celui qu'il écrit à dom Humbert (un moine de Clairvaux, frère uniquement spirituel) mort quelques années plus tard ${ }^{64}$. Saint Bernard évoque davantage les possibilités d'immixtion et de superposition entre parenté charnelle et spirituelle que leur concurrence. Le lien charnel est présenté dans ces exemples comme une qualité supplémentaire, qui augmente la proximité spirituelle. La parenté spirituelle peut inclure la parenté charnelle sans se limiter à celle-ci. « Le sang, comme chacun sait est plus épais que l'eau / Mais l'eau est plus vaste, grâce à Dieu, que le sang ${ }^{65}$. »

\section{NOTES}

1. "Adhaesit anima mea animae illius ; et unam fecit de duabus, non consanguinitas, sed unanimitas. Carnis quidem necessitudo non defuit; sed plus iunxit societas spiritus, consensus animorum, morum conformitas », BERNARD DE CLAIRVAUX, Sermons sur le cantique, tome 2, 26, 9. Texte latin par J. LECLERCQ, H. ROCHAIS et CH. H. TALBOT (sources chrétiennes, 431), Paris, 1998, p. 300 (traduction personnelle).

2..A. BARBERO, Un santo in famiglia, vocazione religiosa e resistenze sociali nell'agiografia latina medievale, Turin, 1991, p. 217.

3..Pierre Bonte définit comme pseudo-parenté des types de « relations sociales qui s'expriment en termes de parenté (de référence ou d'adresse) sans pour autant résulter de liens de parenté effectivement reconnus (par la consanguinité ou le mariage) », P. BONTE, « Pseudo-parenté » dans P. BONTE et M. IZARD dir., Dictionnaire de l'ethnologie et de l'anthropologie, Paris, 1991, p. 550.

4..A. GUERREAU-JALABERT, « Spiritus et caritas. Le baptême dans la société médiévale », dans F. HÉRITIER et E. COPET-ROUGIER dir., La Parenté spirituelle, Paris, 1994, p. 133-203.

5..Sur ce sujet voir S. D'ONOFRIO, L'Esprit de la parenté : Europe et horizon chrétien, préface de F. HÉRITIER, Paris, 2004, et A. FINE, Parrains, marraines. La parenté spirituelle en Europe, Paris, 1994.

6..Peu d'études traitent spécifiquement de la concurrence entre parenté monastique et parenté charnelle. Hormis les analyses d'Alessandro Barbero déjà citées, on peut renvoyer à A. BORIAS, « Le moine et sa famille ", Collectanea Cisterciensia 40 (1978), p. 81-110 et p. 195-217 ; à titre de comparaison, on peut consulter une étude récente d'anthropologie contemporaine A. HERROU, La Vie entre soi. Les moines taoïstes aujourd'hui en Chine, Nanterre, 2005.

7..B. DE CLAIRVAUX, Sermons sur le Cantique, texte latin par J. LECLERCQ, H. ROCHAIS et CH. H. TALBOT, Paris, (Sources chrétiennes 431), 1998.

8..PL 185, col. 225-368.

9..Vita Secunda, PL 185, col. 469-554. 
10..Jacques de Voragine dans la Légende Dorée résume surtout la Vita prima, par l'intermédiaire de l'abréviation de Jean de Mailly, cf. IACOPO DA VARAZZE, Legenda Aurea, edizione critica a cura di Giovanni Paolo Maggioni, seconda edizione rivista dall'autore, Florence, 1998.

11. Voir J.-C. SCHMITT, « Le culte de Saint Bernard et ses images », dans Saint Bernard et le monde cistercien, L. PRESSOUYNE et T. N. KINDER (ed.), Paris, 1990, p. 149-163 ; ID, « Saint Bernard et son image », dans Bernard de Clairvaux. Histoire, mentalités, spiritualité ; Colloque de Lyon-Cîteaux-Dijon, Paris, (Sources chrétiennes 380), 1992, p. 639-657.

12..Voir J. BERLIOZ « La lactation de saint Bernard dans un exemplum et une miniature du Ci nous dit (début du XIV ${ }^{\text {e }}$ siècle) », dans Cîteaux, Commentarii cistercienses, 39, 1988, p. 270-284, ainsi que C. DUPEUX, «La lactation de saint Bernard, genèse et évolution d'une image ", dans L'image et la production du sacré, actes du colloque de Strasbourg, (20-21 janvier 1988) organisé par le centre d'Histoire des religions de l'Université de Strasbourg II, F. DUNANT, J. SPIESER, et J. WIRTH dir., Paris, 1991, p. 165-193.

13..Sur l'apport de l'iconographie à l'étude des représentations de la parenté spirituelle voir en particulier : J. BASCHET, Le sein du père, Abraham et la paternité dans l'Occident Médiéval, Paris, 2000 et ID., « La parenté partagée : engendrement charnel et infusion de l'âme (à propos d'une miniature de la fin du XV siècle) ", dans Anima e Corpo nelle cultura medievale, Atti del V Convegno di studi della società italiana per lo studio del pensiero Medievale, Venezia 25-28 settembre 1995, Florence, 1999, p. 123-137.

14..Voir J. MARILIER, Histoire de l'Église en Bourgogne, Dijon, 1991 ; A. H. BREDERO, Bernard de Clairvaux. Culte et Histoire, Turnhout, 1998 ; A. LOUF, « Bernard abbé », dans Bernard de Clairvaux, Histoire, mentalités..., op. cit., p. 349-379 ; J. BERLIOZ, Saint Bernard en Bourgogne, Dijon, 1990.

15..Voir D. LETT, « L'allaitement des saints au Moyen Âge. Un seul sein vénérable : le sein de la Vierge », dans Allaitements en marge, D. BONNET, C. LE GRAND-SÉBILLE et M.-F. MOREL dir., Paris, 2002, p. 163-174.

16..« Propter quod etiam alienis uberibus nutriendos committere illustris femina refugiebat, quasi cum lacte materno materni quodammodo boni infundens eis naturam », GUILLAUME DE SAINT-THIERRY, Vita prima Bernardi claravallensis, livre I, chapitre 1 paragraphe 1, PL 185, col. 225-368.

17..« Siquidem ab illa hora, sicut ignis qui comburit silvam, et sicut flamma comburens montes, hinc inde prius viciniora quaeque corripiens, postmodum in ulteriora progrediens : sic ignis quem miserat Dominus in cor servi sui volens ut arderet, primo fratres ejus aggreditur, solo minimo ad conversionem adhuc minus habili, seniori patri ad solatium derelicto, deinde cognatos, et socios, et amicos, de quibuscunque poterat esse spes conversionis », ibid., livre 1 chapitre III, paragraphe 10, PL 185, col. 232.

18. « Jamque eo publice et privatim praedicante, matres filios abscondebant, uxores detinebant maritos, amici amicos avertebant ; quia voci ejus Spiritus Vita prima », PL 235D.

19..Rejet appuyé par les références évangéliques : « qui aime son père ou sa mère plus que moi n'est pas digne de moi, qui aime son fils ou sa fille plus que moi n'est pas digne de moi » (Mt, 10, 35-37), qui se marque souvent par un changement de nom, et le don de sa part d'héritage au monastère. Cette situation, abandonner la parenté de chair pour la parenté monastique, est fondatrice de nombreux modèles cénobitiques et érémitiques hérités de l'antiquité tardive, comme dans la vie de saint Martin, $c f$. A. BARBERO, op. cit., chapitre 1 .

20..R. FOSSIER, « L'installation et les premières années à Clairvaux (1115-1119) », dans Bernard de Clairvaux, Histoire, mentalités... op. cit., p. 87.

21..Liber ad milites Templi de laude novae militiae édité dans : B. DE CLAIRVAUX, Vie de - Éloge de la nouvelle chevalerie Épitaphe, hymne, lettres, Euvres complètes XXXI - saint Malachie, Introductions, traductions, notes et index par P.-Y. EMERY, Paris, (Sources chrétiennes 367), 1990.

22..Voir note 10

23..« Guido primogenitus fratrum suorum Nivardum fratrem suum minimum, puerum cum pueris aliis in platea : "Eia ", inquit, “ frater Nivarde, ad te solum respicit omnis terra possessionis nostrae." Ad quod puer 
non pueriliter motus : "Vobis ergo ", inquit, “ coelum, et mihi terra ? Non ex aequo divisio haec facta est." Quo dicto abeuntibus illis, tunc quidem domi cum patre remansit, sed modico post evoluto tempore fratres secutus, nec a patre, nec a propinquis seu amicis potuit retineri. Supererat de Deo dicata domo illa pater senior cum filia, de quibus etiam suo loco dicemus », Vita prima, chapitre 3, paragraphe 17, PL 185, col. 232-233.

24..Au tout début du quinzième siècle, Jean Gerson réitère en quelque sorte l'image de la famille spiritualisée voulue par Bernard de Clairvaux, à ceci près qu'il était l'aîné de sa fratrie, et donc mieux placé pour donner des conseils et recommandations. Dans une lettre adressée à ses sœurs pour les engager à rester vierges et à s'occuper de leurs parents, il leur explique que leurs autres frères, devenus clercs ou moines, leur laisseront à disposition tout l'héritage parental pour leur permettre de subvenir à leurs besoins, voir $\mathrm{F}$.

LEDWIDGE, « Relations de famille dans la correspondance de Gerson », Revue Historique, 271, 1984, p. 1-23, et

B. P. MCGUIRE, « Late medieval care and control of women : Jean Gerson and his sisters », Revue d'histoire ecclésiastique, 92, 1997, p. 5-37.

25..Joseph Morsel évoque à propos de la dévalorisation des liens charnels et des transferts opérés de la parenté de chair vers la parenté spirituelle une « déparentalisation » et plus récemment une «transparentalisation ». Le second terme me semble particulièrement adapté au cas de saint Bernard car il n'exclut pas que la parenté spirituelle soit une véritable parenté, ni que des entrelacements puissent avoir lieu entre les deux, J. MORSEL (avec la collaboration de C. DUCOURTIEUX), L'Histoire (du Moyen Âge) est un sport de combat... Réflexions sur les finalités de l'Histoire du Moyen Âge destinées à une société dans laquelle même les étudiants d'Histoire s'interrogent, LAMOP-Paris I, 2007, p. 129.

26..« Egressus est de domo paterna Bernardus, pater fratrum suorum, cum fratribus suis, filiis suis spiritualibus, quos verbo vitae Christo genuerat », Vita prima, 3, 17, PL 185, col. 236.

27..À ce sujet, voir les analyses de J. BASCHET, Le Sein du père... op. cit., p. 56.

28..B. DE CLAIRVAUX, Lettre 1, 10, (Sources chrétiennes 425), Paris, 1997.

29..Sermons sur le cantique, 23, 2. Sur ces métaphores, voir C. BYNUM, « Jesus as Mother and Abbot as Mother : Some themes in Twelfth-Century Cistercian Writing ", dans Jesus as Mother : Studies in the Spirituality of the High Middle Ages, Berkeley, 1982, 110-168. Cependant, dans la continuité des études de Jérôme Baschet, l'on ne peut réduire les métaphores d'engendrement à une simple expression féminine. L'engendrement, le sein, restent pour Dieu comme pour Abraham, des images attribuées au masculin.

30..« Je remplacerai auprès de lui son père et sa mère, son frère et sa sœur ", écrit-il en consolation aux parents du moine Geoffroy dans la Lettre 110, 2.

31..Présence quantitative des termes de parenté primaire (déclinés) : frater/fraternus : 84, germanus/a: 3 , soror : 2 , mater $: 25$, pater $: 31$, filius/filia $: 38$, maritos $: 1$, uxor $: 6$, sponsus $: 1$, parens $: 3$, mares $: 2$.

32..Cette étude lexicale a été réalisée dans le cadre d'une thèse de doctorat en préparation à l'EHESS : Parenté, hagiographie et images chez Jacques de Voragine, c. 1265-1490, sous la direction de Jean-Claude Schmitt.

33..Voir par exemple les images de certains manuscrits de la Légende Dorée du XIV ${ }^{\mathrm{e}}$ siècle, comme le Biblioteca Apostolica Vaticana, Reg. Lat. 534 f. 152 (vers 1300), ou BNF, Fr. 241, f. 214 (daté de 1348) ou encore la célèbre et plus tardive illustration de Jean Fouquet du Musée Condé Le Livre d'Heures d'Étienne Chevalier, Ms. 71, f. 40 (c. 1450).

34..La représentation des ordres monastiques selon une structure arborée, analogique à celle des arbres de parenté est fréquente mais n'existe cependant pas chez les Cisterciens, voir D. DONADIEU-RIGAUT, Penser en images les ordres religieux (XII ${ }^{-}-X V^{e}$ siècles), Paris, 2005, p. 258.

35..Les panneaux latéraux sont consacrés à saint Benoît et saint Jean l'Évangéliste à gauche, et à saint Quentin et saint Galgano à droite.

36..Au début du treizième siècle, Conrad d'Eberbach décrit en effet cette guérison comme un des nombreux miracles accordés à Bernard en précisant : «Comment le seigneur aurait-il méprisé son très humble serviteur 
qui plaidait sa cause avec un cœur brisé alors qu'il l'exauçait avec autant de facilité que de bénignité quand il le priait pour les autres? ", dans Le grand Exorde de Cîteaux, ou récit des débuts de l'ordre cistercien, traduit du latin par A. PIEBOURG, J. BERLIOZ dir., Turnhout, Cîteaux-Commentarii cistercienses, 1998, p. 132.

37..PL 178, col. 1865, passage traduit dans l'Introduction de BERNARD DE CLAIRVAUX, Sermons sur le Cantique, II, Sermons 16-32, op. cit., p. 27.

38..« Quid mihi et cantico huic, qui in amaritudine sum ? Vis doloris abducit intentionem [...]», ibid, Sermon $26,18,3$.

39..« Frater erat genere, sed religione germanior », ibid., 4.

40.« Amarissima separatio, et quam non posset omnino efficere, nisi mors! Quando enim me vivus vivum desereres? Omnno opus mortis, horrendum divortium. Quis enim tam suavi vinculo mutui nostri non pepercit amoris, nisi totius suavitatis inimical mors ? Bene mors quae unum rapiendo, duos furiosa peremit. Annon mors etiam mihi ? ", ibid., 4.

41..« Scis ubi verser, ubi iaceam, sibi reliquere me : non est qui porrigat manum », ibid., 6.

42..Le grand exorde de Citeaux... op. cit., partie 3, chapitre 2.

43..«Deus, inquit, tu scis quoad quantum in me fuit, semper optavi quietem mihi intendere, vacare tibi. Sed implicitum tenuit timor tuus, et voluntas fratrum, et studium oboediendi, super omnia abbatis pariter et fratris germana dilectio ", Sermons sur le Cantique..., op. cit., 26, 6.

44.." Scientibus ista loquor, et adhuc, plura his de illo maiora compertis. Parco tamen, quia caro mea et frater est ", ibid., 7.

45..« Girardum lugeo : Girardus in causa est, frater carne, sed proximus spiritu, socius proposito. Adhaesit anima mea animae illius; et unam fecit de duabus, non consanguinitas, sed unanimitas. Carnis quidem necessitudo non defuit ; sed plus iunxit societas spiritus, consensus animorum, morum conformitas », ibid., 9. 46..C'est une thématique que l'on retrouvera dans la correspondance de Jean Gerson. Le culte que ce dernier voue à Joseph et à la sainte famille va de pair avec sa volonté de façonner sa famille à cette image. Ses parents devaient atteindre un haut degré de spiritualité, et les autres devaient embrasser la vie mystique. Il eut d'ailleurs peu de considération pour la seule de ses sœurs qui s'était mariée. Voir F. LEDWIDGE, art. cit., p. 20. 47..« Meus Gerardus erat, meus plane. An non meus, qui frater sanguine fuit, professione filius, sollicitudine pater, consors spiritu, intimus affectu? Is recessit a me : sentio, laesus sum, et graviter. Ignoscite filii, immo, si filii, vicem dolete paternam », Sermons sur le Cantique..., op. cit., sermon 26, 9-10.

48. Voir FR. AVRIL et N. REYNAUD, Les Manuscrits à peinture en France 1440-1520, Paris, 1993, notice 138 p. 258 Le Miroir Historial est attribué à Maître François, artiste parisien jusque dans les années 1480, alors que le BNF, fr. 244-245 fut sans doute commencé par le même artiste (folios 1-4) avant d'être repris par l'héritier de son atelier, que l'on nomme le maître de Jacques de Besançon. Il est très possible que l'image de la Légende dorée ait été inspirée par celle du Miroir historial, bien qu'elles diffèrent quelque peu par leur iconographie.

49..Dominique Donadieu-Rigaut, dans une étude sérielle d'images de prises d'habit, en particulier de saint Benoît, relève combien cette scène est le lieu privilégié de la prise de rôle symbolique du père (celui qui confère le vêtement). D. DONADIEU-RIGAUT, Penser en images les ordres religieux..., op. cit., p.102.

50..Par exemple la Sainte parenté du Maître de la Sainte Parenté, Cologne, Wallraf-Richartz-Museum, ou dans le manuscrit déjà cité BNF fr. 244-245, fol. 84 ; sur ce thème voir J. WIRTH, L'image médiévale. Naissance et développements ( $\mathrm{VI}^{\mathrm{e}-\mathrm{XV}} \mathrm{V}^{\mathrm{e}}$ siècle), Paris, 1989, p. 307-308.

51..Voir Eros Philadelphe, Frère et sœur, passion secrète, colloque de Cérisy, W. BANNOUR et P. BERTHIER (dir.) Paris, 1992.

52. « Soror quoque eorum in saeculo nupta, et saeculo dedita, cum in divitiis saeculi periclitaretur, tandem aliquando inspiravit ei Deus ut fratres suos visitaret. Cumque venisset quasi visura venerabilem fratrem suum, et adesset cum comitatu superbo et apparatu, ille detestans et exsecrans eam tanquam rete diaboli ad capiendas animas, nullatenus acquievit exire ad videndum eam. Quod audiens illa, confusa, et compuncta 
vehementer, cum ei nullus fratrum suorum occurrere dignaretur, cum a fratre suo Andrea, quem ad portam invenerat monasterii, ob vestium apparatum stercus involutum argueretur, tota in lacrymas resoluta ", Vita prima, 6, PL. 185, Col. 244.

53..« Illa vero optata libertate potita, monasterium Julleium adiens, cum sanctimonialibus inibi Deo servientibus reliquum vitae suae Deo vovit : ubi tantam ei Dominus gratiam contulit sanctitatis, ut non minus animo quam carne illorum probaretur virorum Dei esse germana », Vita prima, PL 185, col. 245

54..C'est la même remarque que l'on peut faire pour Jean Gerson au quinzième siècle, qui ne veut pas de couvent pour ses sœurs à qui il recommande de rester vierges ensemble dans la maison familiale. Une seule d'entre elles finit par se marier contre l'avis de son frère, F. LEDWIDGE, loc. cit., et B.P MCGUIRE, loc. cit. 55..«Quia enim peccatrix sum, idcirco consilium et colloquium bonorum requiro. Et si despicit frater meus carnem meam, ne despiciat servus Dei animam meam », Ibid, PL 185, col. 242.

56..Composition de la sainte écriture, ou le « Ci nous dit ", Chantilly, musée Condé, ms. 26. Le texte fut écrit dans les années 1313-1330 par un auteur anonyme, Christian Heck date le manuscrit des environs de 1330. Selon François Avril, l'enlumineur pourraît être Mahiet, que l'on identifie avec Matthieu le Vavasseur, clerc normand, qui dirigeait un atelier à Paris vers 1330-1350, C. HECK, « Description et mémoire du texte dans les enluminures du "Ci nous dit" ", Le Texte de l'œuvre d'art, Strasbourg, 1998, p. 19-31.

57..Sur ce manuscrit commandé par la cour des Anjou-Hongrois et enluminé dans un style bolonais, voir B. ZSOLT SZAKÁCS, « Le culte des saints à la cour et le Légendier des Anjou-Hongrie », dans L'Europe des Anjou (catalogue de l'exposition à l'abbaye de Fontevraud), Paris, 2001, 195-201.

58. « Cum enim aliquando curiosius aspiciendo defixos in quam dam oculos aliquamdiu tenuisset, continuo ad se reversus, et de semetipso reubescens apud semetipsum, in seipsum ultor severissimus insiliens, tamdiu inibi permansit, donec pene exsanguis effectus, per virtutem gratiae cooperantis etiam a calore carnalis concupiscentiae totus refriguit », Vita prima, III, 6, PL 185, col. 230.

59..Transcription de la légende : « II Q(uo)m(odo) iacebat $\mathrm{cu}(\mathrm{m})$ sorore sua. Et occulte surgens posuit se i(n) aq(ua)m ut fornicat(io) s(ibi) n(on) eve(n)it. »

60..L'inceste adelphique consommé ou non est un motif récurrent dans l'hagiographie médiévale, et sa prohibition par les clercs comme un crime terrible est fréquente et répétée, parfois moteur d'un choix de conversion, A. GUERREAU-JALABERT, « Grégoire ou le double inceste, le rôle de la parenté comme enjeu (XII ${ }^{\mathrm{e}}$ $\mathrm{XIX}^{\mathrm{e}}$ siècle) ", dans Réception et identification du conte depuis le Moyen Âge, textes réunis par M. ZINK et X. RAVIER, Toulouse, 1987, p. 21-38 et D. LETT, Histoire des frères et sœurs, Paris, 2004, p. 198.

61..« Andreas Bernardo dixit : “Ecce, date operam ne quis fratrum nostrorum in saeculo maneat : alioquin scinde medium, quoniam nec a vobis, nec ab illis separari tolerabile est" ", Vita tertia, III, PL 185, col. 525.

62..R. JAMOUS, La Relation frère-sœur, parenté et rites chez les Meo de l'Inde du Nord, Paris, 1991, p. 225.

63..F. HÉRITIER, L'Exercice de la parenté, Paris, EHESS, 1980, p. 171.

64..«Tu m'as pris mes frères selon la chair, qui l'étaient plus encore par l'esprit, et qui étaient sages à tes yeux aussi bien pour tes intérêts que pour les affaires de ce monde. Tu les as retirés d'ici les uns après les autres, eux qui portaient mon fardeau, ce fardeau très pesant que tu m'as imposé. De tous ceux qui m'étaient si précieux, il m'en restait un, presque seul, Humbert, ami d'autant plus cher qu'il était plus ancien, et tu l'as pris parce qu'il était à toi. Je reste seul devant l'adversité, je meurs moi-même en chacun d'eux, et tu as fait déferler sur moi tous les flots. " In obitu domni Humberti, cité dans Le grand exorde de Cîteaux..., op. cit., p. 140.

65..« Blood, as all men know, than water's thicker / But water's wider, thank the Lord, than blood ", A. HUXLEY, Leda, "Ninth Philosopher's Song”, dans Verse and a comedy, Early poems, Leda, The Cicadas, the world of light, London, 1946, Dans cet aphorisme, Huxley détourne le sens du proverbe bien connu des Anglo-saxons, « Blood is thicker than Water », sur lequel s'appuya David Schneider pour critiquer l'anthropologie de la parenté dans A Critique of the Study of Kinship, Ann Arbor, 1984. 


\section{RÉSUMÉS}

L'entrée en monachisme implique, selon l'idéal ascétique, d'abandonner sa fratrie biologique pour une nouvelle fratrie soudée par des liens spirituels. L'étude proposée convoque l'exemple de Bernard de Clairvaux (1090-1153), qui convertit l'ensemble de ses frères charnels (qui le suivirent à Clairvaux) et sa sœur (qui devint moniale au couvent bénédictin de Jully). Il nous présente ainsi un cas singulier dans lequel parenté charnelle et parenté spirituelle parviennent, au prix de conflits et de conversions fermement orchestrés par le futur saint, à se superposer exactement. Impliqué personnellement par le problème de définition du lien adelphique, il présente dans un sermon sur le Cantique des cantiques consacré à la mort de son frère Gérard une définition de la hiérarchie entre les liens de parenté. Le cas de saint Bernard, symptomatique de la réorganisation des représentations de la parenté au temps du monachisme réformé, devint exemplaire et fut la source d'images figurant la parenté spirituelle au XIve et $\mathrm{XV}^{\mathrm{e}}$ siècle.

Bernard of Clairvaux and recomposed sibship. According to ascetic ideals, becoming a monk implies relinquishing one's carnal brotherhood for a spiritual one. The present paper presents the example of Bernard of Clairvaux (1090-1153), who converted all his brothers (who followed him to Clairvaux) and his sister (who became a nun in the Benedictine monastery of Jully). The conversions were some time conflictual, but they allowed the future saint to make his carnal and spiritual kinship coincide. This even lead him to propose a definition of the hierarchy between the different bounds of kinship in a famous sermon on the Song of Songs, dedicated to his brother Gerard's death. The example of Bernard can be read as a symptom of how the representation of kinship was reorganised in the time of Reformed monachism. It was also used as a model for images representing spiritual kinship in the XIvth and xvth centuries.

\section{INDEX}

Mots-clés : Bernard de Clairvaux, cisterciens, fratrie, iconographie, parenté spirituelle

Keywords : Bernard of Clairvaux, Cistercians, iconography, sibship, spiritual kinship

\section{AUTEUR}

CHLOÉ MAILLET

EHESS/GAHOM, 2 rue Vivienne, F-75002 Paris 